\title{
Gut Microbiota Changes and Their Relationship with Inflammation in Patients with Acute and Chronic Insomnia
}

This article was published in the following Dove Press journal: Nature and Science of Sleep

\author{
Yuanyuan $\mathrm{Li},{ }^{1,2, *}$ \\ Bin Zhang, $\mathbb{D}^{3, *}$ Ya Zhou, ${ }^{4}$ \\ Daoming Wang, (iD) \\ Xianchen Liu, ${ }^{1,6}$ Lin Li, ' \\ Tong Wang,' Yuechu Zhang,' \\ Min Jiang,' Huilan Tang, ${ }^{7}$ \\ Lawrence $\vee$ Amsel, ${ }^{7}$ \\ Fang Fan,' Christina W Hoven ${ }^{7}$
}

'Key Laboratory of Brain, Cognition and Education Sciences, Ministry of Education, China; School of Psychology, Center for Studies of Psychological Application, and Guangdong Key Laboratory of Mental Health and Cognitive Science, South China Normal University, Guangzhou, People's Republic of China; ${ }^{2}$ Expressive Arts Therapy Department, Sun Yat-sen Memorial Hospital, Sun Yat-sen University, Guangzhou, People's Republic of China; ${ }^{3}$ Department of Psychiatry, Nanfang Hospital, Southern Medical University, Guangzhou, People's Republic of China; ${ }^{4}$ Department of Psychology, Lund University, Lund, Sweden; ${ }^{5}$ School of Future Technology, University of Chinese Academy of Sciences, Beijing, People's Republic of China; ${ }^{6}$ The University of Tennessee Health Science Center, Memphis, TN, USA; ${ }^{7}$ Department of Child and Adolescent Psychiatry, New York State Psychiatric Institute, Columbia University, New York, NY, USA

*These authors contributed equally to this work

Correspondence: Fang Fan School of Psychology, Center for Studies of Psychological Application, South China Normal University, Guangzhou 5I063I, People's Republic of China Email fangfan@scnu.edu.cn
Purpose: The major purpose of this study was to detect the changes in gut microbiota composition and inflammatory cytokines production associated with acute and chronic insomnia. This study also evaluated the relationship between gut microbiota changes and increased inflammatory cytokines in insomnia patients.

Patients and Methods: Outpatients with acute and chronic insomnia (aged 26-55 years; $\mathrm{n}=20$ and 38 , respectively) and age/gender-matched healthy controls $(\mathrm{n}=38)$ were recruited from a southern China region. Participants' gut microbiome, plasma cytokines, and selfreported sleep quality and psychopathological symptoms were measured.

Results: The gut microbiomes of insomnia patients compared with healthy controls were characterized by lower microbial richness and diversity, depletion of anaerobes, and shortchain fatty acid (SCFA)-producing bacteria, and an expansion of potential pathobionts. Lachnospira and Bacteroides were signature bacteria for distinguishing acute insomnia patients from healthy controls, while Faecalibacterium and Blautia were signature bacteria for distinguishing chronic insomnia patients from healthy controls. Acute/chronic insomniarelated signature bacteria also showed correlations with these patients' self-reported sleep quality and plasma IL-1 $\beta$.

Conclusion: These findings suggest that insomnia symptomology, gut microbiota, and inflammation may be interrelated in complex ways. Gut microbiota may serve as an important indicator for auxiliary diagnosis of insomnia and provide possible new therapeutic targets in the field of sleep disorders.

Keywords: acute insomnia, chronic insomnia, gut microbiome, inflammatory cytokines, random forest

\section{Introduction}

Insomnia is the most prevalent sleep disorder affecting about $10-50 \%$ of the adult population worldwide, ${ }^{1-3}$ which is predominantly characterized by difficulty initiating or maintaining sleep, poor sleep quality, and impaired daytime functioning. ${ }^{4}$ Psychiatric disorders such as anxiety, depression, bipolar disorder, and schizophrenia, as well as a broad range of physical disorders, particularly metabolic-related disorders such as obesity and type 2 diabetes, are also frequent among patients with insomnia. 5,6

Many studies have suggested the etiology of insomnia may be related to many factors such as genetics, biochemistry, neuroendocrine, immune, and psychosocial factors. ${ }^{7}$ In recent years, increasing evidence has identified the microbiome-gutbrain axis, with gut microbiome central to the multi-directional effects on brain 
and physiology through immune, hormonal, and neural pathways. ${ }^{8-10}$ Gut microbiome has been shown to be associated with sleep. For example, in a rodent model, interfering with the sleep patterns can change the structure and diversity of intestinal microbiota. ${ }^{11}$ Similar changes in microbial community structure have been observed in shift workers who have insufficient sleep. ${ }^{12}$ Moreover, treatment of intestinal microbiota imbalance has the effect to improve sleep duration and sleep efficiency. ${ }^{13}$ Previous research suggests that systematic inflammation may be a pathway linking gut microbiome with sleep. Insomnia symptoms and chronic sleep restrictions have been shown to increase the production of inflammatory cytokines such as IL- $1 \beta$ and TNF- $\alpha .^{14,15}$ Increased gut microbiome diversity may also cause a reduction in circulating pro-inflammatory cytokines, which may in turn improve sleep. ${ }^{13}$

However, most of these studies were conducted in animal models. Very few studies have examined in human samples how the gut microbiome is associated with insomnia symptomology and psychological impairments and inflammatory cytokine changes. To fill this gap of knowledge, the present study examined and compared the composition of gut microbiota and production of inflammatory cytokines between acute and chronic insomnia patients and healthy subjects. The major purpose was to detect the changes in gut microbiota composition and inflammatory cytokines production associated with acute and chronic insomnia. As in the literature, ${ }^{14,16}$ patients with acute and chronic insomnia compared with healthy subjects were hypothesized to have increased inflammatory cytokines and gut microbiota disturbance. This study also attempted to evaluate the relationship between gut microbiota changes and increased inflammatory cytokines in insomnia patients.

\section{Participants and Methods}

\section{Participants}

Insomnia patients between 26-55 years were recruited from outpatients in the department of Psychiatry of Nanfang Hospital in Guangzhou, Guangdong, from May 2017 to December 2018. The Mini-International Neuropsychiatric Interview was used as a systematic psychiatric screening tool to detect pre-existing psychiatric disorders. ${ }^{17}$ The Structured Clinical Interview for the Diagnostic and Statistical Manual of Mental Disorders-Fifth Edition was used to diagnose acute or chronic insomnia disorder. ${ }^{18}$
Healthy subjects (age-, sex-, and body mass index-matched with the patients) who resided in the same geographic area and had similar dietary habits were recruited through advertisements and screened using a semi-structured clinical interview to exclude those with psychiatric or physical illnesses. To ensure the homogeneity of different groups of participants, the following exclusion criteria were applied: hypertension; cardiovascular disease; diabetes mellitus; obesity; liver cirrhosis; fatty liver disease; irritable bowel syndrome; inflammatory bowel disease; other sleep disorders; drug or alcohol abuse in the last 6 months; use of antibiotics, probiotics, prebiotics, symbiotic, or acid-suppressive drugs in the month before the fecal sample collection; known active bacterial, fungal, or viral infections; and have jet lag or shift work experience in the month before collection of the samples. Table 1 presents the demographic and psychosocial characteristics of the study participants.

All participants were examined clinically after recruitment by two psychiatrists and were subsequently divided into three groups: acute insomnia disorder (AID) $(n=20)$, chronic insomnia disorder (CID) group $(n=38)$, and healthy controls $(\mathrm{HC})(\mathrm{n}=38)$. The AID patients were defined as having insomnia longer than 1 week but shorter than 3 months. Persons with CID were defined as those having insomnia longer than 3 months. This study was conducted in accordance with the Declaration of Helsinki and was approved by the Human Research Ethics Committee of South China Normal University. Written informed consent was provided by all participants before data collection.

\section{Questionnaires}

Participants' sleep quality and sleep disturbance during the past month was assessed by Pittsburgh Sleep Quality Index (PSQI). ${ }^{19}$ General mental health status was measured by Generalized Anxiety Disorder 7-item (GAD-7) scale $^{20}$ and Patient Health Questionnaire 9-item (PHQ-9) scale. ${ }^{21}$ Four items about abdominal dysfunction extracted from the Patient Health Questionnaire (PHQ-15) ${ }^{22}$ were used to assess abdominal dysfunction.

A Self-designed Dietary Assessment Scale was used to assess dietary habits, which includes 12 self-designed items mainly developed from the Adult Eating Behavior Questionnaire. ${ }^{23}$ This scale was divided into three dimensions: regular diet (eating meals at regular times), healthy diet (frequency of high-calorie special diet intake), and rich diversity in diet (general food intake frequency of grains, vegetables, fruits, milk, beans, egg, fish, and 
meat). The respondent rated each item on a 5-point scale, ranging from 1 ("never") to 5 ("always"). A summary score (range $=12-60$ ) was calculated by adding all items together, so that higher scores reflect a greater degree of regular diet, healthy diet, and rich diversity in diet.

\section{Plasma Cytokine Detection}

Blood samples were provided by the 96 participants in the morning (08:00 am-11:00 am). Samples were collected in an ethylenediamine tetraacetic acid (EDTA)-containing tube then centrifuged for 10 minutes at $1500 \mathrm{rpm}, 4^{\circ} \mathrm{C}$. Plasma was aliquoted into cryotubes and stored at $-80^{\circ} \mathrm{C}$ until assayed. Plasma tumor necrosis factor- $\alpha$ (TNF- $\alpha$ ), interleukin-1 $\beta$ (IL-1 $\beta$ ), and IL-6 were measured using commercially available enzyme-linked immunosorbent assay kits (ELISA kits of TNF- $\alpha$, IL-1 $\beta$, IL- 6 were purchased from Neobioscience Technology Co., Ltd.).

\section{Fecal Sample Collection and DNA Extraction}

Fecal samples were collected in the morning (08:00 am11:00 am) within 3 days after the completion of the questionnaires and were kept in iceboxes ( $4^{\circ}$ Celsius). Fecal microbial DNA was extracted from $200 \mathrm{mg}$ feces using the HiPure Stool DNA Kits B (D3141-03B, Guangzhou meiji biotechnology co., Ltd, China) according to the manufacturer's instructions.

\section{Quantitative Polymerase Chain Reaction Analysis for Bacteria}

We performed 16S rRNA sequencing as described before. ${ }^{24}$ Briefly, the V3-V4 region of the bacterial 16S rRNA gene was amplified by PCR using the following primer pair: 341F, CCTACGGGNGGCWGCAG, and 806R, GGACTACHVGGGTATCTAAT, and quantified using an ABI StepOnePlus Real-Time PCR System (Life Technologies, USA) was performed. Library quantification, normalization, pooling, and denaturation were performed as per manufacturer's protocol (Illumina, San Diego, CA, USA). Samples were sequenced on a Hiseq 2500 platform and using a PE250 mode.

\section{Statistical Analysis and Bioinformatics}

Statistical analyses were performed using the SPSS software program (version 23.0) (IBM Corp., Armonk, NY), Mplus 8.3 and R 3.6.1. Differences in demographics and inflammatory cytokines were analyzed using the analysis of variance
(ANOVA) or multivariate analyses of covariance (MANCOVA), followed by Fisher's least significant difference post hoc test.

The raw tags obtained from the sequencer were demultiplexed and quality-filtered using FLASH (v 1.2.11) to obtain clean tags. ${ }^{25}$ Chimera sequences arising from the PCR amplification were detected and removed using UCHIME Algorithm, and all remaining sequences were assigned to samples according to the barcodes and clustered into operational taxonomic units (OTUs) using Uparse (usearch v9.2.64_i86linux32) ${ }^{26}$ with a $97 \%$ threshold of pairwise identity. OTUs that reached a $97 \%$ nucleotide similarity level were used for rarefaction curve analyses using Mothur. Alpha diversity was measured using QIIME based on the level of OTUs, Chao estimate, observed species (Sobs), abundance-based coverage estimator (ACE), Shannon and Simpson index. Beta diversity was estimated by Bray-Curtis dissimilarity and by computing UniFrac distances that were reported by principal coordinate analysis (PCoA). Using the Kruskal-Wallis rank-sum test, and linear regression model controlled for covariates, we further accessed the difference in microbiome features among acute and chronic insomnia, and healthy controls. To identify the key signature microbiota, 5-fold cross-validation together with Random Forest were performed to compute importance scores (mean decrease accuracy, MDA) to estimate the importance of variables by utilizing the "random Forest" v.4.6-14 package in R 3.6.1. ${ }^{27}$ The operating characteristic curves (receiving operational curve, ROC) were constructed and area under curve (AUC) was calculated to assess the diagnostic performance of the model with the pROC 1.15 .3 package. ${ }^{28}$ Partial Spearman's rank-based correlation was employed to investigate the correlation between inflammatory cytokines and the signature bacteria for insomnia in CID and AID group, respectively. Statistical significance was set at $P<0.05$.

\section{Results}

\section{Sample Characteristics}

The whole sample consisted of 96 participants $(\mathrm{AID}=20$; $\mathrm{CID}=38 ; \mathrm{HC}=38$ ). Table 1 presents the demographics and psychosocial characteristics of the sample.

ANOVA revealed that the AID and CID groups had significantly poorer self-reported sleep quality, more depressive and anxious symptoms, worse marital status, and more abdominal dysfunction symptoms compared to HCs. All three groups had similar dietary habits. Neither of them regularly consumed alcohol within the previous month, and only two participants regularly used tobacco products. 
Table I Demographics, Psychosocial Characteristics, Dietary Habits, and Blood Inflammation Markers of the Sample (N=96)

\begin{tabular}{|c|c|c|c|c|}
\hline Variables & AID $(n=20)$ & $\operatorname{CID}(n=38)$ & HC $(n=38)$ & $\mathbf{F} / \chi^{2}$ \\
\hline \multicolumn{5}{|l|}{ Demographics } \\
\hline Age, years, mean $\pm S D$ & $37.80 \pm 7.40^{\# \#}$ & $43.47 \pm 6.86 * *$ & $38.50 \pm 7.05$ & 6.35 \\
\hline Gender, Female, n (\%) & 15 (75.0\%) & $25(65.8 \%)$ & 18 (47.4\%) & 4.94 \\
\hline $\mathrm{BMI}, \mathrm{kg} / \mathrm{m}^{2}$, mean $\pm \mathrm{SD}$ & $21.79 \pm 1.95$ & $22.21 \pm 2.43$ & $22.82 \pm 1.97$ & 1.65 \\
\hline Education level, n (\%) & & & & 13.64 \\
\hline Below high school & $9(45 \%)$ & 27 (7I.1\%) & II (28.9\%) & \\
\hline Above high school & II (55\%) & II (28.9\%) & 27 (7I.1\%) & \\
\hline Marital Status, n (\%) & & & & 10.92 \\
\hline Poor & $9(45 \%)$ & 18 (47.4\%) & $6(18.2 \%)$ & \\
\hline Good & $8(40.0 \%)$ & 17 (44.7\%) & $28(73.7 \%)$ & \\
\hline Single & $3(15.0 \%)$ & $3(7.9 \%)$ & $4(10.5 \%)$ & \\
\hline Personal monthly income, $\mathrm{n}$ (\%) & & & & 6.92 \\
\hline$>¥ 10,000$ & II (55.0\%) & $22(57.9 \%)$ & $19(50.0 \%)$ & \\
\hline$¥ 5,000-Y 10,000$ & $9(45.0 \%)$ & II (28.9\%) & $10(26.3 \%)$ & \\
\hline$<¥ 5,000$ & $0(0.0 \%)$ & 5 (13.2\%) & $9(23.7 \%)$ & \\
\hline Regular Exercise & & & & 0.39 \\
\hline Yes & $10(50.0 \%)$ & 22 (57.9\%) & $20(52.6 \%)$ & \\
\hline No & $10(50.0 \%)$ & $16(42.1 \%)$ & 18 (47.4\%) & \\
\hline \multicolumn{5}{|c|}{ Sleep/Emotional Disorder and Somatic Symptoms, mean \pm SD } \\
\hline $\mathrm{PSQ}^{\mathrm{a}}$ & $14.85 \pm 3.23 * * *$ & $16.26 \pm 3.68^{* * *}$ & $3.00 \pm 1.4 \mid$ & 105.06 \\
\hline PHQ & $8.95 \pm 4.52 * * *$ & $9.82 \pm 4.40 * * *$ & $2.79 \pm 2.59$ & 36.00 \\
\hline GAD & $6.95 \pm 4.88^{* * *}$ & $7.87 \pm 4.73 * * *$ & $2.34 \pm 2.82$ & $|8.7|$ \\
\hline Abdominal dysfunction ${ }^{a}$ & $8.5 I \pm 2.66$ & $10.06 \pm 3.62 * *$ & $5.98 \pm 2.00$ & 3.82 \\
\hline \multicolumn{5}{|l|}{ Dietary habits, mean $\pm S D$} \\
\hline Regular diet & $8.10 \pm 1.36$ & $7.09 \pm 1.85$ & $7.12 \pm 2.37$ & 1.95 \\
\hline Healthy diet & $20.67 \pm 2.21$ & $19.88 \pm 2.24$ & $19.95 \pm 2.06$ & 0.97 \\
\hline Rich diversity in diet & $12.49 \pm 2.05$ & $11.56 \pm 1.47$ & $12.28 \pm 1.65$ & 2.68 \\
\hline \multicolumn{5}{|c|}{ Blood inflammation marker, ${ }^{b}$ mean \pm SD } \\
\hline $\mathrm{IL}-\mathrm{I} \beta(\mathrm{pg} / \mathrm{mL})$ & $21.56 \pm 18.59 * *$ & $16.73 \pm 8.65^{*}$ & $11.07 \pm 10.05$ & 5.25 \\
\hline IL-6 (pg/mL) & $11.47 \pm 11.39$ & $20.30 \pm 20.39$ & $|3.52 \pm| 7.4 \mid$ & 2.35 \\
\hline TNF- $\alpha(p g / m L)$ & $17.91 \pm 7.10$ & $23.19 \pm 11.97$ & $17.56 \pm 10.11$ & 2.16 \\
\hline
\end{tabular}

Notes: ${ }^{\mathrm{a} C}$ Controlling for PHQ and GAD score. ${ }^{\mathrm{b}}$ Results from MANCOVA with Blood inflammation markers as dependent variable, group as fixed factor and age, gender, BMI, PHQ and GAD score as covariates. Superscript symbols indicate statistically significant differences between the following: $* * * P<0.001, * * P<0.01, * P<0.05$, each group with respect to $\mathrm{HC}$ group; ${ }^{\#} \mathrm{P}<0.0 \mathrm{I}$, each group with respect to the CID group. Abdominal dysfunction was measured by self-designed abdominal dysfunction questionnaire (4 items about abdominal dysfunction extracted from Patient Health Questionnaire, PHQ-15).

Abbreviations: PSQI, Pittsburgh Sleep Quality Index score; PHQ-9, Patient Health Questionnaire 9-item; GAD, Generalized Anxiety Disorder 7-item (GAD-7) scale; IL-Iß, interleukin-I beta; IL-6, interleukin-6; TNF- $\alpha$, tumor necrosis alpha; AID, acute insomnia disorder; CID, chronic insomnia disorder; HC, healthy controls.

\section{Decreased Alpha-Diversity and Altered} Overall Microbial Composition in Insomnia Based on the OTUs, various alpha-diversity indexes (ie, Chao estimate, observed species [Sobs], abundance-based coverage estimator [ACE], Shannon and Simpson index) were used to evaluate the ecological features of the fecal bacterial community in the sample. The CID group compared with the HCs showed a significantly decreased intraindividual diversity, as indicated by the Chaol index $(P=0.017)$. Though the fecal microbial diversity estimated using the $\operatorname{ACE}(P=0.056)$ and Sobs $(P=0.051)$ Index was also somewhat lower in the CID group than in HCs, the difference was not statically significant (Wilcoxon rank-sum test; Figure 1A-C). The trend in alphadiversity in patients with AID was similar to that of the HCs, based on the Chao $(P=0.065)$, ACE $(P=0.135)$, and Sobs $(P=0.104)$ index, respectively (Wilcoxon rank-sum test; 
Figure 1A-C). There were no significant differences in the Shannon or Simpson index among the three groups (Supplementary Figure S1A and B).

Bacterial beta-diversity was assessed through conducting the principal coordinate analysis based on Bray-Curtis dissimilarity. Permutational multivariate analysis of variance demonstrated significant differences in gut taxonomic composition among the three groups (AID vs $\mathrm{HC}$ : $P=0.001$, CID vs HC: $P=0.001$, AID vs CID: $P=0.001$; Figure 1D). The microbial community among the three groups was also significantly different for weighted UniFrac distance and unweighted UniFrac distance (Supplementary Figure S1C and D).

\section{Altered Gut Microbiota Composition in Insomnia Patients}

The fecal microbiota composition of the sample showed significant differences at multiple taxonomical levels by linear regression model controlled for age, gender, PHQ, GAD, and abdominal dysfunction. Firmicutes, Bacteroidetes, and Actinobacteria were the three most dominant phyla across all three groups. At the phylum level, compared with the HCs, Actinobacteria $(P=0.0005)$ were over-represented in the CID group, whereas Firmicutes $(P=0.011)$ were significantly decreased in the AID group (Supplementary Figure S2).

At the genus level, significant differences were also observed among the three groups. There were five bacterial taxa showing distinct relative abundances between the CID and HC groups $(P<0.05)$; specifically, decreased abundance in Faecalibacterium, Prevotella 9, and Roseburia, and increased abundance in Blautia and Eubacterium hallii were observed in the CID group (Figure 2). In the AID group, Bacteroides was increased, whereas Lachnospira were decreased compared with HCs (Figure 2). There was no difference among the three groups at species level.

In total, the CID/AID groups were different from the $\mathrm{HCs}$ in the relative abundances of seven genera.

\section{Signature Bacteria for Insomnia}

To identify signature bacteria that could distinguish acute and chronic insomnia patients from healthy controls, we incorporated a robust statistical analysis and applied 5-fold crossvalidation together with random forest to create a classification model based on the abovementioned seven genera, with consideration of lowest error rate and standard deviation. We found that the combination of two genera including Lachnospira and Bacteroides were most useful for distinguishing acute insomnia patients and healthy controls, with an AUC of 87.5\% (95\% CI=78.7-96.3\%) (Figure 3A and D). The combination of two genera, Faecalibacterium, and Blautia could distinguish chronic insomnia patients from healthy controls, with an AUC of $92.97 \% \quad(95 \%$ $\mathrm{CI}=87.16-98.79 \%$ ) (Figure 3B and D).

\section{Inflammatory Cytokines in Insomnia}

As shown in Table 1, results of MANCOVA (controlling for age, gender, BMI, PHQ, and GAD scores) showed that both AID $(P<0.01)$ and CID $(P<0.05)$ groups had significantly increased levels of IL- $1 \beta$ than HCs. Although the CID group also had an increased level of IL- 6 and TNF- $\alpha$ than the HC and AID group, the difference is not significant (Table 1).

\section{Signature Bacteria Associated with Inflammatory Cytokines in Insomnia}

Partial Spearman's rank-based correlation tests (controlling for age, gender, PHQ, GAD, and abdominal dysfunction scores) were employed to investigate the associations between acute/chronic insomnia patients' signature bacteria and their inflammatory cytokines (IL-1 $\beta, \mathrm{TNF}-\alpha$ ), and selfreported sleep quality (measured by PSQI). We found that in the CID group, the combination of two signature bacteria Faecalibacterium, and Blautia (CID probability) was significantly correlated with PSQI $(\mathrm{r}=0.49, P<0.001)$, and IL-1 $\beta$ $(\mathrm{r}=0.45, P<0.001)$ (Figure 4A). In the AID group, the combination of two signature bacteria Lachnospira, and Bacteroides (AID probability) was positively correlated with PSQI ( $\mathrm{r}=0.48, P<0.001$ ) (Figure 4B).

With regard to specific signature bacteria, in the AID group, lower abundance of Lachnospira ( $r=-0.48$, $P<0.001)$, and higher abundance of Bacteroides $(r=0.35, P=0.01)$ were significantly correlated with higher PSQI score (Figure 4A). In the CID group, lower abundance of Faecalibacterium was significantly correlated with a higher plasma level of IL-1 $\beta(r=-0.27$, $P=0.02)$ and higher scores on PSQI $(r=-0.43, P<0.001)$, whereas higher abundance of Blautia was significantly correlated with a higher plasma level of IL-1 $\beta(r=0.58$, $P<0.001)$ and higher scores on PSQI $(r=0.40, P<0.001)$ (Figure 4B).

\section{Discussion}

There is growing evidence that alterations in the intestinal microbiome correlate with many mental disorders and 

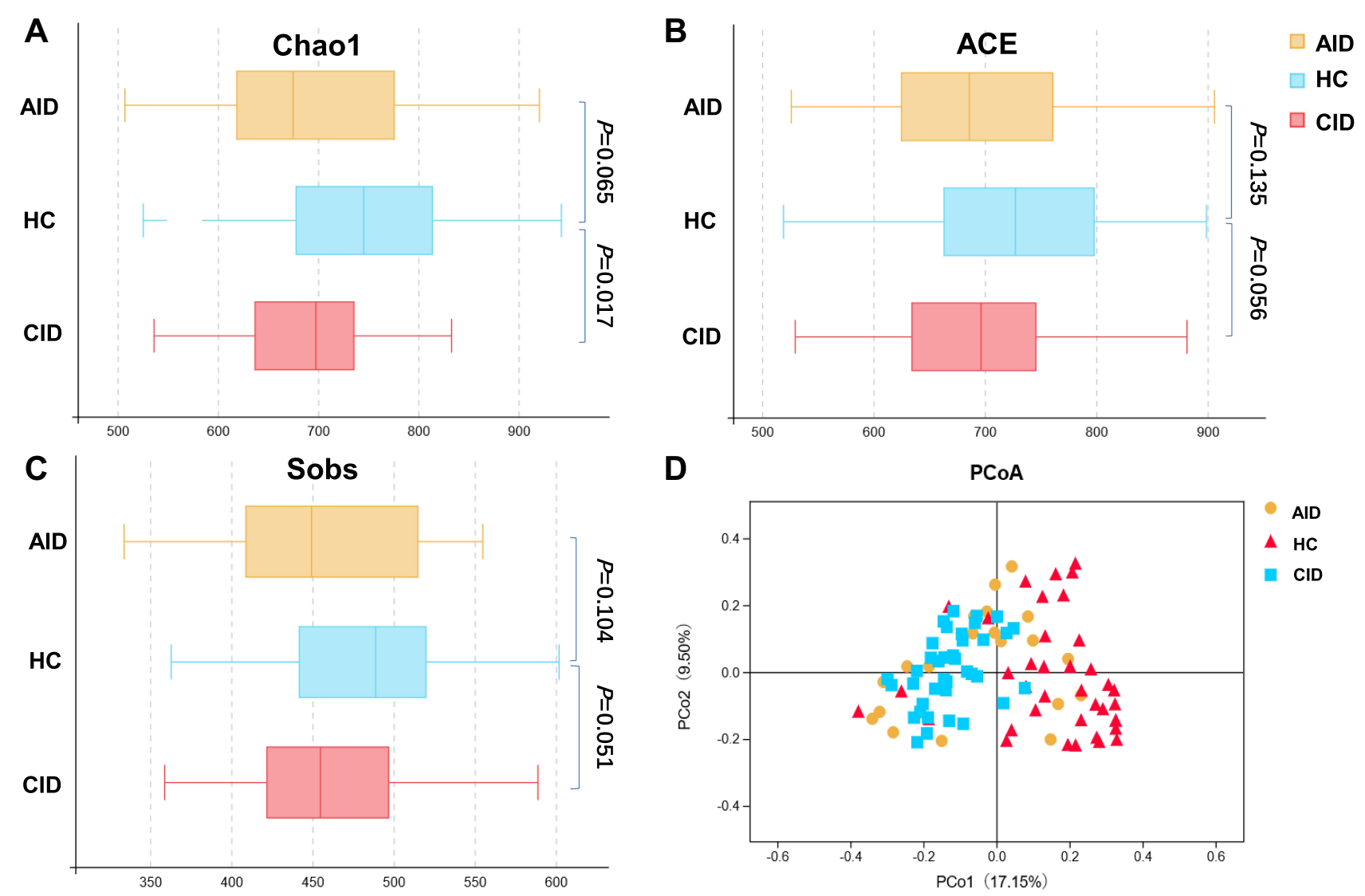

D

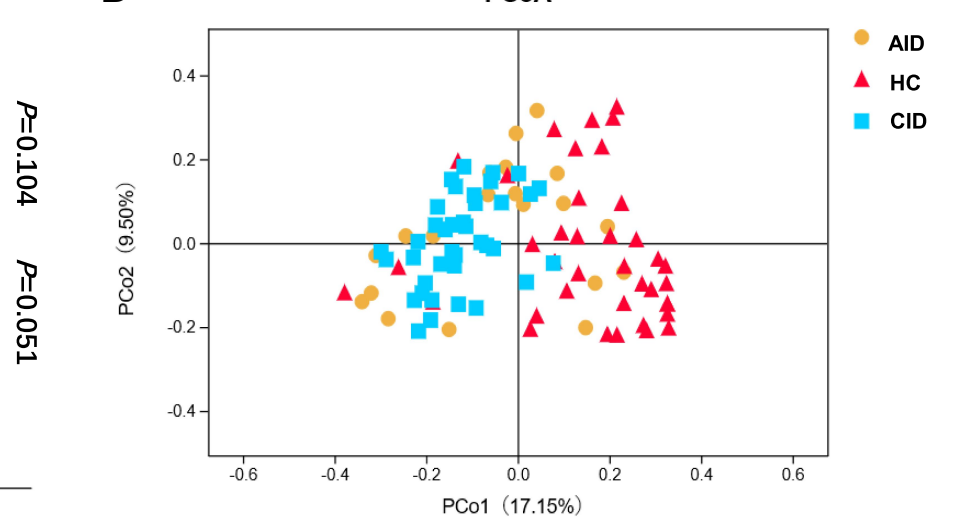

Figure I Phylogenetic diversity of gut microbiomes among AID, CID, and HC groups.

Notes: Comparison of the Chao $(\mathbf{A})$, ACE $(\mathbf{B})$, and sobs $(\mathbf{C})$ index between different groups (CID vs HC: $P=0.017, P=0.056$, and $P=0.05$ I, respectively; $A I D$ vs HC: $P=0.065$, $P=0.135$, and $P=0.104$, respectively; Wilcoxon rank-sum test). (D) $P C o A$ of Bray-Curtis dissimilarity analysis demonstrated that the overall fecal microbiome composition of AID and CID clustered each separately from HCs, and the microbiome composition was also different between AID and CID (AID vs CID vs HC: P<0.00I).

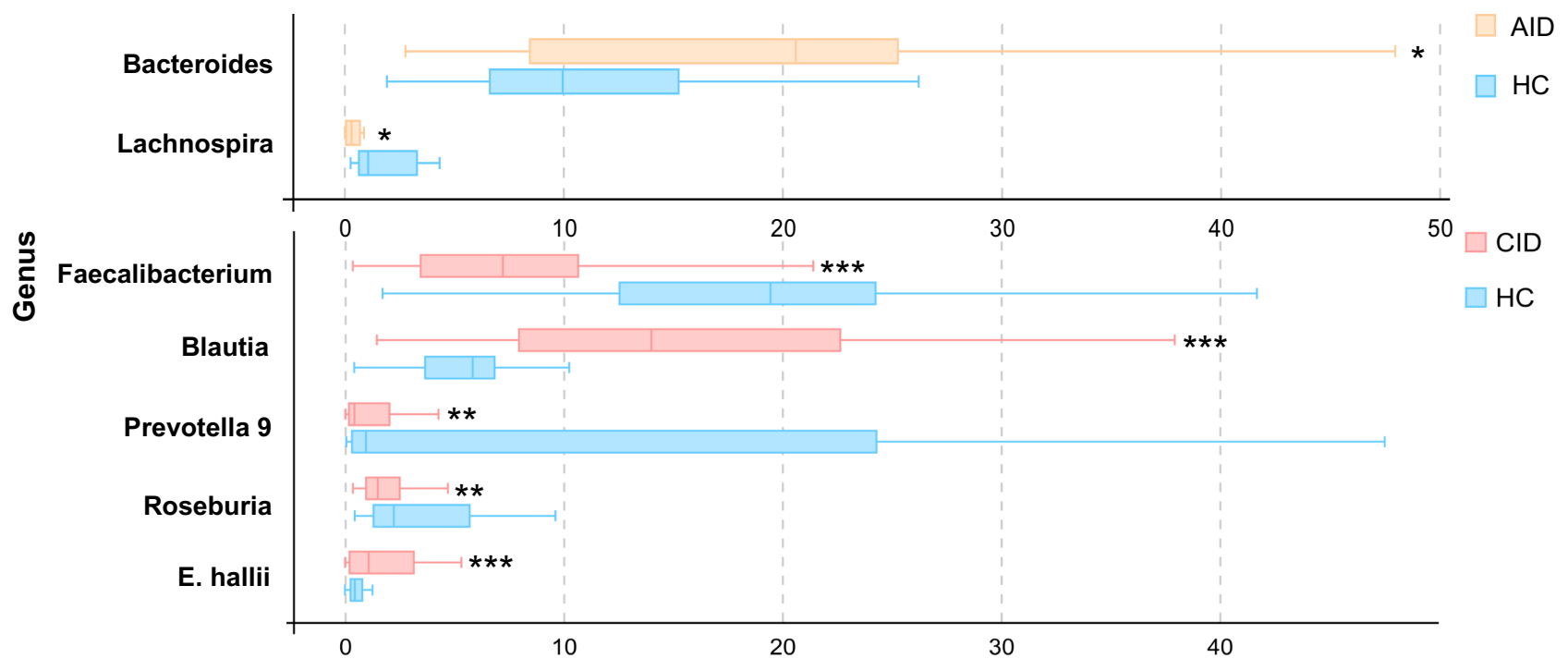

Figure 2 Comparison of relative abundance (>I\% of the total sequences in either group) at the bacterial genus level between AID, CID, and HC groups.

Notes: Linear regression model controlled for age, gender, PHQ, GAD, and abdominal dysfunction. $* P<0.05, * * P<0.01$, $* * * P<0.00$ I, each group with respect to the HC group. 


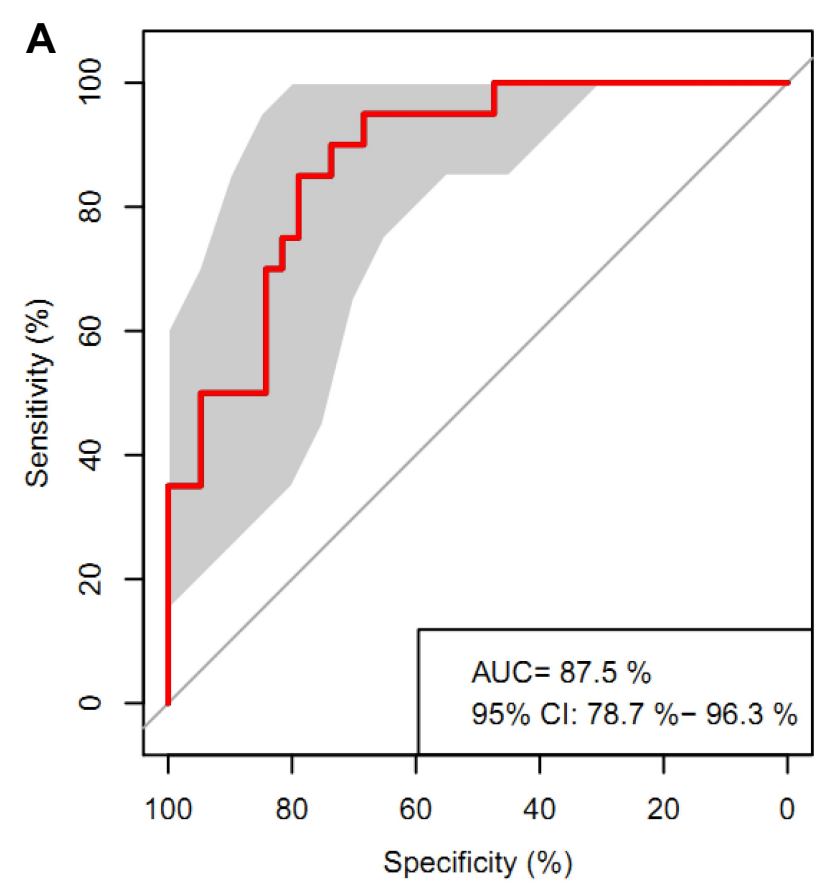

C

\section{Mean Decrease Accuracy}

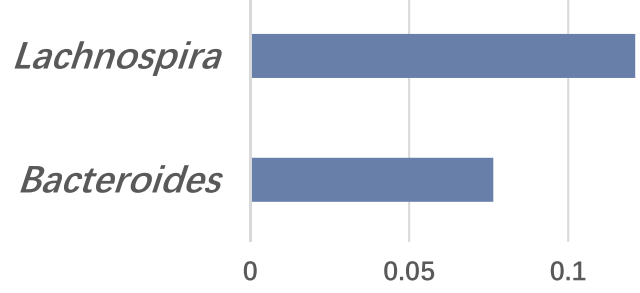

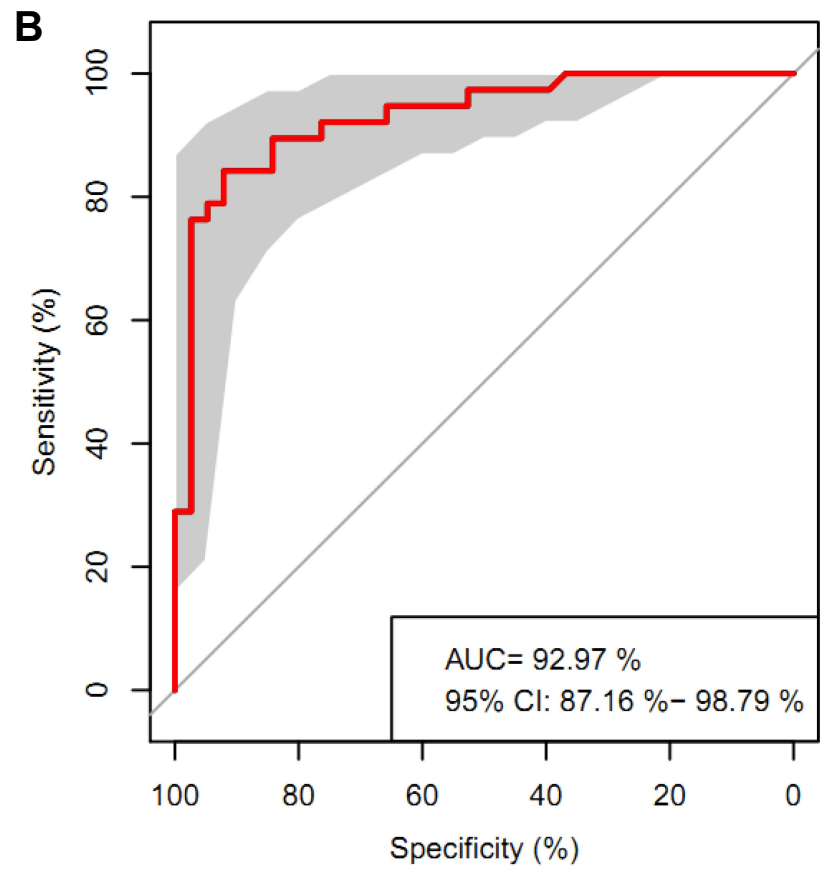

Mean Decrease Accuracy

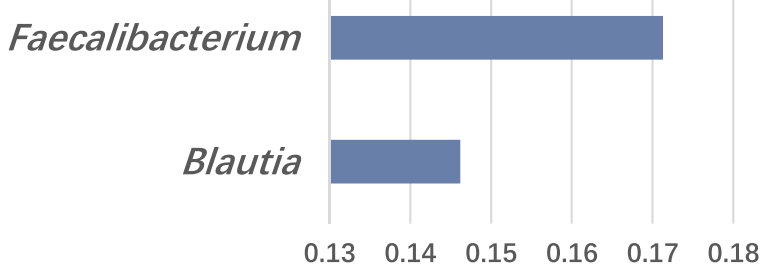

Figure 3 Disease classification based on gut microbiome signature. (A and B) Classification performance of random forest model using relative abundance of insomniaassociated genera was assessed by area under the ROC in AID patients (A) and CID patients (B), respectively. (C and D) Identification of the signature gut microbiota associated with insomnia disorder by random forest. To identify the signature biomarkers, 5 -fold cross-validation together with random forest was performed. (C) The detailed results of random forest in signature biomarkers' random seed are presented between AID and HC group; (D) The detailed results of random forest in signature biomarkers' random seed are presented between CID and HC group.

Notes: AID, acute insomnia disorder, $n=20$; CID, chronic insomnia disorder, $n=38$; HC, healthy controls, $n=38$; ROC, receiving operational curve.

with disrupted physiological homeostasis. ${ }^{29,30}$ However, much remains unclear about the relationship between gut microbiome and human insomnia and related inflammatory cytokine changes. The current study is the first to address such issues in human patients with acute and chronic insomnia.

Insomnia is a common sleep disorder and it has been clearly associated with different aspects of psychosocial dysfunctions such as increased emotional or behavioral symptoms, diminished subjective well-being perceived life quality, and worsened social skills. ${ }^{31}$ The cause of insomnia is thought to involve a complex interaction of many factors. One important factor may be pro-inflammatory activation. Chronic sleep restriction has been shown to be associated with elevated brain IL-1 $\beta$ and TNF- $\alpha,{ }^{15}$ and increased daytime plasma IL-6 concentration. $^{32}$ Another study examined sleep duration and inflammatory biomarkers of 614 individuals, and found that habitual sleep durations were positively associated with IL-6 level. ${ }^{16}$ The current study also found that both AID and CID patients had notably higher plasma level of IL-1 $\beta$ than healthy controls. CID patients were also found to have higher plasma level of TNF- $\alpha$ and IL- 6 than healthy controls and AID patients, although the difference is not significant. These imply that pro-inflammatory cytokine levels may increase with greater severity of insomnia symptomology and IL-1 $\beta$ is an important clinical indicator.

Low bacterial diversity, an important indicator of gut dysbiosis, has been documented in a variety of psychophysiological diseases, including insomnia, depression, and anxiety. ${ }^{33,34}$ In the current study, the CID group compared 
A

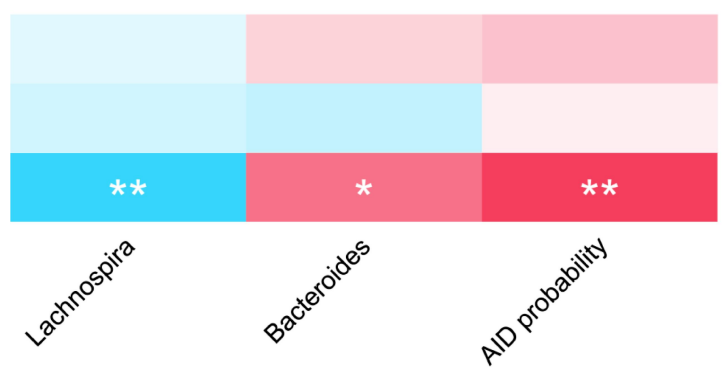

B

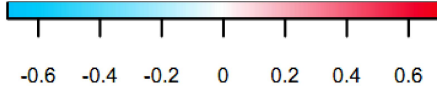

Spearman's correlation coefficient

IL-1 $\beta$
TNF- $\alpha$
PSQI

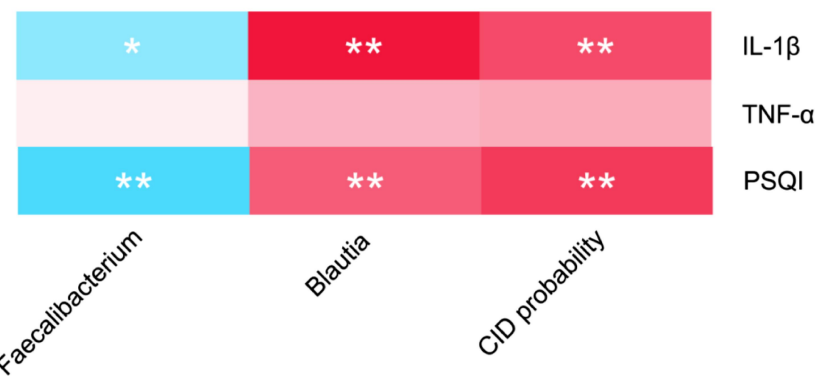

Figure 4 (A) Partial correlation between relative abundance of signature gut microbiota and insomnia-related inflammatory cytokines by partial Spearman's rank-based correlation test in AID and HC group. (B) Partial correlation between relative abundance of signature gut microbiota and insomnia-related inflammatory cytokines by partial Spearman's rank-based correlation test in CID and $\mathrm{HC}$ group.

Notes: $(\mathbf{A}) * P<0.05, * * P<0.01$.

Abbreviations: PSQI, Pittsburgh Sleep Quality Index; IL-I $\beta$, interleukin-I $\beta$; TNF- $\alpha$, tumor necrosis factor- $\alpha$; AID probability, combination of Lachnospira and Bacteroides; CID probability, combination of Faecalibacterium and Blautia.

with HCs showed a significantly lower alpha-diversity (estimated using the Chao1 Index) in microbial community, while the difference between AID group and HCs did not reach significance, suggesting that microbiome diversity varies in different stages of insomnia pathology, longer duration of insomnia may have a bigger impact on the patients' microbiome diversity. This result was also consistent with one prior study, which reported that patients with CID had significantly altered $\alpha$-diversity of the gut microbiota that characterized by decreased Chaol index. ${ }^{35}$ However, although the microbiota diversity was somewhat greater in HCs, no significant differences within species richness and evenness estimates by estimators such as the ACE, Sobs, Shannon, and Simpson indices were found among the three groups. This may be that the difference in species richness of the samples is more obvious, especially for low-abundance species, but the species evenness of the sample may be similar.

Regarding relative abundances in microbiota composition, AID and CID patients showed decreased abundance in many anaerobic intestinal microbiota such as genera in Lachnospira, Roseburia, and Prevotella 9, indicating that their microbial community may shift towards depletion of anaerobic microbes. The decreased abundances of these genera in insomnia patients may reduce short-chain fatty acid (SCFA) production in their gut, which is known to have anti-inflammatory effects and is beneficial to health. $^{36,37}$ Among CID patients, we also observed decreased abundance in Faecalibacterium, Prevotella 9, and Roseburia and increased abundance in Blautia, and Eubacterium hallii, but these were not shown in AID patients, suggesting that microbiome structure may vary in different stages of insomnia pathology. In addition, as the CID group showed decreased abundance in these butyrate producers (eg, Roseburia, Faecalibacterium), ${ }^{38}$ and decreased butyrate is associated with increases in inflammatory markers, ${ }^{39}$ which may also explain the elevated inflammation level in chronic insomnia patients.

Our results from the random forest found that two genera Blautia and Faecalibacterium were most useful for distinguishing CID patients from HCs. Blautia is a common gut inhabitant that belongs to the family Lachnospiraceae. The microbiome is highly plastic and can alter rapidly in response to diet. But previous research also indicates that hostspecificity and host-preference patterns of organisms within Blautia are driven by host physiology more than dietary habits. ${ }^{40}$ Our data also found that higher abundance of Blautia may contribute to the higher plasma level of IL-1 $\beta$ in CID patients. There have been mixed findings about the role of Blautia genus in inflammation, ie, it may be positively $^{41,42}$ or negatively ${ }^{43,44}$ associated with inflammatory dysregulation. The discrepancy among these findings may be due to the different age, BMI, life stages, and pathological circumstances across the study sample. ${ }^{42}$ As for Faecalibacterium, decreased Faecalibacterium representation has been linked with several diseases including bipolar disorder, ${ }^{45}$ depression, ${ }^{33}$ anxiety, ${ }^{34}$ Parkinson's disease, Type 2 diabetes, and ulcerative colitis. ${ }^{46}$ Murine models have found that sleep deprivation significantly decreased the content of Faecalibacterium. ${ }^{47}$ Animal experiments have also reported that Faecalibacterium can enhance goblet cell 
differentiation, leading to an increase in goblet cell numbers and mucin gene expression, ${ }^{48}$ which would reverse the intestinal barrier dysfunction resulting from interactions between gut microbiota dysbiosis and mucosal injury. Previous findings, together with our results, suggest that Blautia and Faecalibacterium may have a close relationship with inflammation, which may further affect chronic insomnia and insomnia-related health decline.

Random forest together with the cross-validation model also found Lachnospira and Bacteroides were the most important biomarkers to discriminate AID patients from HCs. Previous studies have reported the associations between these taxa and psychopathology. ${ }^{34,49}$ A recent study comparing the $16 \mathrm{~S}$ rDNA genes of gut microbiome composition in 10 chronic insomnia patients and 10 healthy controls also found that Bacteroidetes (increased) could be used as one critical biomarker to identify chronic insomnia disorder. $^{35}$ The reason why our results were not completely consistent with their findings may be because we used a larger and demographically more homogeneous sample.

We also found a significant correlation between the combination of Lachnospira and Bacteroides and AID patients' poor self-reported sleep quality, but the correlation with AID patients' cytokine levels was not significant. As stated earlier, the signature biomarkers of chronic insomnia (ie, combination of Faecalibacterium, and Blautia) showed significant correlations with CID patients' poor self-reported sleep quality and with their IL-1 $\beta$ level. This may be because AID patients have a relatively short period of insomnia, and gut microbiota dysbiosis during a relatively short period may not be sufficient to trigger the inflammatory change. One previous study has also reported that the neuroendocrine dysregulation was more expressed in patients with CID than patients with AID, and this may be a contributing factor in maintaining disturbed sleep. ${ }^{50}$ However, to our knowledge there is no study that has examined the mechanism of the difference in inflammatory changes between AID and CID patients, and more research is needed to bridge this important gap.

Methodologically, the current study has several advantages such as a large sample size, inclusion of insomnia patients in different pathological stages (ie, acute and chronic insomnia), utilization of the cutting edge bioinformatic algorithm which incorporated machine learning approaches such as random forest to identify signature bacteria for acute and chronic insomnia and explore their links with insomnia-related emotional and inflammatory impairments. Nevertheless, there are some limitations of the current study and the findings should be considered with caution. First, this was a single center study, which may limit the generation of the microbiota-based diagnostic model. Recently, $\mathrm{He}$ et $\mathrm{al}^{51}$ reported that host regional variation had a strong effect on gut microbiota composition. Multicenter studies including subjects from different regions of the world will be required to strengthen the conclusions. Second, although restrict inclusion/exclusion criteria were used to rule in/out participants (ie, all participants had similar dietary habits), some participants might have prescribed medication intake, and there were also some environmental or lifestyle factors that may influence participants' gut microbiota and confound our findings. Third, although informative, this study employed a crosssectional design which could not provide evidence of causality. Longitudinal research is needed to confirm the prediction of signature bacteria on future insomnia and clarify temporal pathways between gut microbiota changes, pro-inflammatory activation, and insomnia pathology.

\section{Conclusion}

In summary, the current study adds to the literature by identifying differential gut microbiota changes in AID and CID patients and revealing the links between these changes and acute/chronic insomnia-related inflammatory impairments. The findings support the importance of including a microbiota-based strategy into auxiliary diagnosis for insomnia, ${ }^{40}$ and inform possible new therapeutic targets aimed at regulating gut microbiota in the field of sleep disorders. Future understanding about the pathological mechanisms of insomnia may consider the role of gut microbiota.

\section{Acknowledgments}

The present study was funded by the National Natural Science Foundation of China (Grant No. 31871129); Project of Key Institute of Humanities and Social Sciences, MOE (grant number: 16JJD190001); Guangdong Province Universities and Colleges Pearl River Scholar Funded Scheme, GDUPS (2016).

We thank our study participants, as well as research assistants Honglei Yin, Yongzhi Zhao, Na Yan, Ting Liu, Xiaoyan Chen, Dingxuan Chen, Haiying Qi, and Yuan Lai for their assistance during the data collection. We also would like to thank Susan Lin for her help in improving the quality of the manuscript. 


\section{Disclosure}

All authors report no conflicts of interest in this work.

\section{References}

1. Mai E, Buysse DJ. Insomnia: prevalence, impact, pathogenesis, differential diagnosis, and evaluation. Sleep Med Clin. 2008;3 (2):167-174. doi:10.1016/j.jsmc.2008.02.001

2. Ohayon MM. Epidemiology of insomnia: what we know and what we still need to learn. Sleep Med Rev. 2002;6(2):97-111. doi:10.1053/ smrv.2002.0186

3. Roth T. Insomnia: definition, prevalence, etiology, and consequences. J Clin Sleep Med. 2007;3(5 Suppl):S7-S10. doi:10.5664/jcsm.26929

4. Morin CM, Drake CL, Harvey AG, et al. Insomnia disorder. Nat Rev Dis Primers. 2015;1:15037. doi:10.1038/nrdp.2015.37

5. Hertenstein E, Feige B, Gmeiner T, et al. Insomnia as a predictor of mental disorders: a systematic review and meta-analysis. Sleep Med Rev. 2019;43:96-105. doi:10.1016/j.smrv.2018.10.006

6. Blank M, Zhang J, Lamers F, Taylor AD, Hickie IB, Merikangas KR. Health correlates of insomnia symptoms and comorbid mental disorders in a nationally representative sample of US adolescents. Sleep. 2015;38(2):197-204. doi:10.5665/sleep.4396

7. Bollu PC, Kaur H. Sleep medicine: insomnia and sleep. Mo Med. 2019;116(1):68-75.

8. Cryan JF, Dinan TG. Mind-altering microorganisms: the impact of the gut microbiota on brain and behaviour. Nat Rev Neurosci. 2012;13(10):701-712. doi:10.1038/nrn3346

9. Macpherson AJ, Harris NL. Interactions between commensal intestinal bacteria and the immune system. Nat Rev Immunol. 2004;4 (6):478-485. doi:10.1038/nri1373

10. Breit S, Kupferberg A, Rogler G, Hasler G. Vagus nerve as modulator of the brain-gut axis in psychiatric and inflammatory disorders. Front Psychiatry. 2018;9:44. doi:10.3389/fpsyt.2018.00044

11. Thaiss CA, Levy M, Korem T, et al. Microbiota diurnal rhythmicity programs host transcriptome oscillations. Cell. 2016;167(6):14951510.e12. doi:10.1016/j.cell.2016.11.003

12. Reynolds AC, Paterson JL, Ferguson SA, Stanley D, Wright KP Jr, Dawson D. The shift work and health research agenda: considering changes in gut microbiota as a pathway linking shift work, sleep loss and circadian misalignment, and metabolic disease. Sleep Med Rev. 2017;34:3-9. doi:10.1016/j.smrv.2016.06.009

13. Jackson ML, Butt H, Ball M, Lewis DP, Bruck D. Sleep quality and the treatment of intestinal microbiota imbalance in chronic fatigue syndrome: a pilot study. Sleep Sci. 2015;8(3):124-133. doi:10.1016/j. slsci.2015.10.001

14. Fernandez-Mendoza J, Baker JH, Vgontzas AN, Gaines J, Liao D, Bixler EO. Insomnia symptoms with objective short sleep duration are associated with systemic inflammation in adolescents. Brain Behav Immun. 2017;61:110-116. doi:10.1016/j.bbi.2016.12.026

15. Zielinski MR, Kim Y, Karpova SA, McCarley RW, Strecker RE, Gerashchenko D. Chronic sleep restriction elevates brain interleukin-1 beta and tumor necrosis factor-alpha and attenuates brain-derived neurotrophic factor expression. Neurosci Lett. 2014;580:27-31. doi:10.1016/j.neulet.2014.07.043

16. Patel SR, Zhu X, Storfer-Isser A, et al. Sleep duration and biomarkers of inflammation. Sleep. 2009;32(2):200-204. doi:10.1093/sleep/ 32.2.200

17. Sheehan DV, Lecrubier Y, Sheehan KH, et al. The mini-international neuropsychiatric interview (M.I.N.I.): the development and validation of a structured diagnostic psychiatric interview for DSM-IV and ICD-10. J Clin Psychiatry. 1998;59(Suppl 20):22-57.

18. American Psychiatric Association. Diagnostic and Statistical Manual of Mental Disorders: Dsm-5; 2013:5
19. Mollayeva T, Thurairajah P, Burton K, Mollayeva S, Shapiro CM, Colantonio A. The Pittsburgh sleep quality index as a screening tool for sleep dysfunction in clinical and non-clinical samples: a systematic review and meta-analysis. Sleep Med Rev. 2016;25:52-73. doi:10.1016/j.smrv.2015.01.009

20. Spitzer RL, Kroenke K, Williams JB, Löwe B. A brief measure for assessing generalized anxiety disorder: the GAD-7. Arch Intern Med. 2006;166(10):1092-1097. doi:10.1001/archinte.166.10.1092

21. Kroenke K, Spitzer RL, Williams JB. The PHQ-9: validity of a brief depression severity measure. J Gen Intern Med. 2001;16(9):606-613. doi:10.1046/j.1525-1497.2001.016009606.x

22. Kroenke K, Spitzer RL, Williams JB. The PHQ-15: validity of a new measure for evaluating the severity of somatic symptoms. Psychosom Med. 2002;64(2):258-266. doi:10.1097/00006842200203000-00008

23. Yi WANG, Sheng W, Guibao ZHU, et al. Method to evaluate an adult eating behavior questionnaire. Chin J Public Health. 2005;21 (2):246-247. doi:10.3321/j.issn:1001-0580.2005.02.072

24. Tang R, Wei Y, Li Y, et al. Gut microbial profile is altered in primary biliary cholangitis and partially restored after UDCA therapy. Gut. 2018;67(3):534-541. doi:10.1136/gutjnl-2016-313332

25. Bokulich NA, Subramanian S, Faith JJ, et al. Quality-filtering vastly improves diversity estimates from Illumina amplicon sequencing. Nat Methods. 2013;10:57. doi:10.1038/nmeth.2276

26. Edgar RC. UPARSE: highly accurate OTU sequences from microbial amplicon reads. Nat Methods. 2013;10:996. doi:10.1038/nmeth.2604

27. Liaw A, Wiener M. Classification and regression by random Forest. R News. 2002;2:18-22.

28. Robin X, Turck N, Hainard A, et al. pROC: an open-source package for $\mathrm{R}$ and $\mathrm{S}+$ to analyze and compare ROC curves. BMC Bioinform. 2011;12:77. doi:10.1186/1471-2105-12-77

29. Lima-Ojeda JM, Rupprecht R, Baghai TC. "I am I and my bacterial circumstances": linking gut microbiome, neurodevelopment, and depression. Front Psychiatry. 2017;8:153. doi:10.3389/fpsyt.2017. 00153

30. Vuong HE, Yano JM, Fung TC, Hsiao EY. The microbiome and host behavior. Annu Rev Neurosci. 2017;40:21-49. doi:10.1146/annurevneuro-072116-031347

31. Thase ME. Correlates and consequences of chronic insomnia. Gen Hosp Psychiatry. 2005;27(2):100-112. doi:10.1016/j.genhosppsych. 2004.09.006

32. Vgontzas AN, Zoumakis M, Papanicolaou DA, et al. Chronic insomnia is associated with a shift of interleukin- 6 and tumor necrosis factor secretion from nighttime to daytime. Metabolism. 2002;51 (7):887-892. doi:10.1053/meta.2002.33357

33. Jiang H, Ling Z, Zhang Y, et al. Altered fecal microbiota composition in patients with major depressive disorder. Brain Behav Immun. 2015;48:186-194. doi:10.1016/j.bbi.2015.03.016

34. Jiang HY, Zhang X, Yu ZH, et al. Altered gut microbiota profile in patients with generalized anxiety disorder. $J$ Psychiatr Res. 2018;104:130-136. doi:10.1016/j.jpsychires.2018.07.007

35. Liu B, Lin W, Chen S, et al. Gut Microbiota as a subjective measurement for auxiliary diagnosis of insomnia disorder. Front Microbiol. 2019;10:1770. doi:10.3389/fmicb.2019.01770

36. Kim MH, Kang SG, Park JH, Yanagisawa M, Kim CH. Short-chain fatty acids activate GPR41 and GPR43 on intestinal epithelial cells to promote inflammatory responses in mice. Gastroenterology. 2013;145(2):396-406.e10. doi:10.1053/j.gastro.2013.04.056

37. Kim CH, Park J, Kim M. Gut microbiota-derived short-chain fatty acids, T cells, and inflammation. Immune Netw. 2014;14(6):277-288. doi:10.4110/in.2014.14.6.277

38. Duncan SH, Barcenilla A, Stewart CS, Pryde SE, Flint HJ. Acetate utilization and butyryl coenzyme A (CoA): acetate-CoA transferase in butyrate-producing bacteria from the human large intestine. Appl Environ Microbiol. 2002;68(10):5186-5190. doi:10.1128/aem.68. 10.5186-5190.2002 
39. Bach Knudsen KE, Lærke HN, Hedemann MS, et al. Impact of diet-modulated butyrate production on intestinal barrier function and inflammation. Nutrients. 2018;10(10):1499. doi:10.3390/nu1 0101499

40. Eren AM, Sogin ML, Morrison HG, et al. A single genus in the gut microbiome reflects host preference and specificity. ISME J. 2015;9 (1):90-100. doi:10.1038/ismej.2014.97

41. Juste C, Kreil DP, Beauvallet C, et al. Bacterial protein signals are associated with Crohn's disease. Gut. 2014;63(10):1566-1577. doi:10.1136/gutjnl-2012-303786

42. Shen F, Zheng RD, Sun XQ, Ding WJ, Wang XY, Fan JG. Gut microbiota dysbiosis in patients with non-alcoholic fatty liver disease. Hepatobiliary Pancreat Dis Int. 2017;16(4):375-381. doi:10.1016/S1499-3872(17)60019-5

43. Zhang J, Guo Z, Xue Z, et al. A phylo-functional core of gut microbiota in healthy young Chinese cohorts across lifestyles, geography and ethnicities. ISME J. 2015;9(9):1979-1990. doi:10.1038/ ismej.2015.11

44. Zhang X, Zhao Y, Xu J, et al. Modulation of gut microbiota by berberine and metformin during the treatment of high-fat diet-induced obesity in rats. Sci Rep. 2015;5:14405. doi:10.1038/ srep 14405

45. Evans SJ, Bassis CM, Hein R, et al. The gut microbiome composition associates with bipolar disorder and illness severity. J Psychiatr Res. 2017;87:23-29. doi:10.1016/j.jpsychires.2016.12.007
46. Tamanai-Shacoori Z, Smida I, Bousarghin L, et al. Roseburia spp.: a marker of health? Future Microbiol. 2017;12:157-170. doi:10.2217/fmb-2016-0130

47. Gao T, Wang Z, Dong Y, et al. Role of melatonin in sleep deprivation-induced intestinal barrier dysfunction in mice. J Pineal Res. 2019;67(1):e12574. doi:10.1111/jpi.12574

48. Wrzosek L, Miquel S, Noordine ML, et al. Bacteroides thetaiotaomicron and Faecalibacterium prausnitzii influence the production of mucus glycans and the development of goblet cells in the colonic epithelium of a gnotobiotic model rodent. BMC Biol. 2013;11:61. doi:10.1186/1741-7007-11-61

49. Poroyko VA, Carreras A, Khalyfa A, et al. Chronic sleep disruption alters gut microbiota, induces systemic and adipose tissue inflammation and insulin resistance in mice. Sci Rep. 2016;6:35405. doi: $10.1038 /$ srep35405

50. Rodenbeck A, Hajak G. Neuroendocrine dysregulation in primary insomnia. Rev Neurol (Paris). 2001;157(11 Pt 2):S57-S61.

51. He Y, Wu W, Zheng HM, et al. Author correction: regional variation limits applications of healthy gut microbiome reference ranges and disease models. Nat Med. 2018;24(12):1940. doi:10.1038/s41591018-0219-z

\section{Publish your work in this journal}

Nature and Science of Sleep is an international, peer-reviewed, open access journal covering all aspects of sleep science and sleep medicine, including the neurophysiology and functions of sleep, the genetics of sleep, sleep and society, biological rhythms, dreaming, sleep disorders and therapy, and strategies to optimize healthy sleep.
The manuscript management system is completely online and includes a very quick and fair peer-review system, which is all easy to use. Visit http://www.dovepress.com/testimonials.php to read real quotes from published authors. 